\title{
A Prediction Model for Initial Trust Formation in Electronic Commerce
}

\author{
Jollean K. Sinclaire \\ Department of Computer and Information Technology \\ Arkansas State University College of Business \\ P.O. Box 130, State University, AR 72467
}

Tel: 011-1-870-972-3990Ｅ-mail: jsinclaire@astate.edu

Judith C. Simon

Department of Management Information Systems

Fogelman College of Business and Economics, University of Memphis

3675 Central Avenue, Memphis, TN 38152

Tel: 011-1-901-678-4613 E-mail: jsimon@memphis.edu

Ronald B. Wilkes

Department of Management Information Systems (Retired)

Fogelman College of Business and Economics, University of Memphis

3675 Central Avenue, Memphis, TN 38152

Tel: 011-1-901-678-4613Ｅ-mail: rbwilkes@memphis.edu

\begin{abstract}
This research investigates trust-building strategies that may influence transactions between individuals and unknown Internet firms, focusing on three influential components that mediate the relationship between online shoppers and online vendors. Results indicate significant direct effects for trust in the Internet infrastructure, susceptibility to the social influence of media, and the presence of influential site characteristics on user willingness to provide personal information to unknown Internet firms. This study extends the research on trust in electronic commerce by providing a prediction model that is demonstrated to calculate the probability of user willingness to provide information. The utility of the model for identifying the relative importance of factors and predicting outcomes lends insight into important issues in online trust formation. Knowledge of effective trust-building strategies guide organizations that use the Internet for selling, marketing, or servicing customers to gain maximum benefits from investments in e-commerce applications.
\end{abstract}

Keywords: Trust, Trusted third parties, Social presence, Social influence, Logistic regression

\section{Introduction}

The increasing rate of data breaches (ITRC, 2009) and the increasing consumer fear of identity theft (Steiner, 2008) indicate a need for guidance on investment in e-commerce applications that meet specific data collection needs of organizations and communicate a credible expression of trustworthiness. Given the current challenging economic environment, it is especially important in the context of initial trust formation that organizations adopt a web strategy that maximizes user confidence yet minimizes investment in the e-commerce application.

Two information processing models offer a theoretical foundation for examining factors that influence online information-giving behavior. The heuristic-systematic model of persuasive communication (Chaiken and Eagly, 1983 ) and the elaboration-likelihood model (ELM) of persuasion (Petty and Cacioppo, 1986) are concerned with changes in attitude as a result of exposure to persuasive messages. Both theories assume that, in the absence of motivation for effortful cognition, individuals process information at a minimal level. Both theories describe cognitive processing as either deep/systematic or shallow/heuristic. Heuristic processing describes a minimizing effort that is more likely to occur when there is limited knowledge, time, or competing demands on cognitive ability (Chaiken, Wood and Eagly, 1996). Systematic processing describes a more effortful process that makes greater demands on cognitive resources (Chaiken, Wood and Eagly, 1996). In the process of initial trust formation, users consider a range of information and utilize a variety of cognitive processing strategies in their decision-making process relating to online information-giving behavior. 
This paper provides a review of prior research, followed by sections describing the research objective, research methodology, and data analyses. The paper concludes with a discussion and conclusion section identifying important findings from this research, along with suggestions for future research.

\section{Background and Literature Review}

A review of research on user willingness to complete a transaction on the Internet reveals common themes of trust in the Internet store (Jarvenpaa, Tractinsky and Vitale, 2000), trust in the vendor (Pennington, Wilcox and Grover, 2003), trust in organizational practices (Smith, Milberg and Burke, 1996), and user perception of Web site features (Belanger, Hiller and Smith, 2002; Gefen and Straub, 2004; Pennington et al., 2003). The literature on trust includes five research streams: personality-based, cognition-based, calculative-based, knowledge-based, and institution-based trust (McKnight, Cummings and Chervany, 1998). Personality-based trust describes trust tendencies that are developed during childhood; cognition-based trust describes trust that develops as a result of first impressions and cues from the environment. Calculative-based trust is based on perceived economic outcomes, and knowledge-based trust occurs as a result of a history of interaction (Gefen, Karahana and Straub, 2003). Institution-based trust is generated by "guarantees, safety nets, or other structures" that convey a sense of security in a situation (Gefen et al., 2003; McKnight et al., 1998).

\subsection{Trust in the Internet Infrastructure}

The Lee and Turban (2001) model of consumer trust in Internet shopping (i.e., trust in the computerized medium) features the perceived technical competence, perceived system performance, and user understanding of the system or the medium. There is evidence of a link between positive perceptions about the trustworthiness of the Internet and Internet purchase intentions (George 2002, 2004), and between institution-based structural assurance and trust-related Internet behaviors (McKnight and Chervany, 2001). Structural assurance is characterized as "technological Internet safeguards" such as encryption (McKnight and Chervany, 2001, p. 5).

\subsection{Web Site Characteristics}

Trusted third parties (TTPs) are organizations that work to reduce consumer fear about online security and privacy and increase trust in e-commerce transactions (Palmer, Bailey, Faraj and Smith, 2000). A TTP acts as a guarantor, providing an assurance of authentication or a brand image or reputation as a foundation for trust. TTPs may be classified according to purpose or intention. Privacy seals represent certified data collection and data usage processes (TrustE, n.d.; BBB, 2010), while security symbols provide assurance that the site uses the secure sockets layer (SSL) cryptographic protocol (GeoTrust, n.d.; VeriSign, 2010). A vulnerability symbol verifies third-party scans for vulnerabilities (HackerSafe, 2010). Reliability symbols vouch for the identity of the Web site and may affirm ethical practices (BBB, 2010; SquareTrade, 2010; WebAssured, n.d.). Consumer rating symbols indicate a satisfied customer experience with the Web site (BizRate, 2009). Although e-commerce literature offers contradictory findings on the ability of TTPs to influence online users, there is evidence of the positive effect of TTPs on purchasing likelihood (Fogg, Soohoo and Danielson, 2002) and information disclosure for some users (Miyazaki and Krishnamurthy, 2002). Additionally, as symbols of expertise, the presence of these artifacts may result in less thought given to scrutiny of information about the Web vendor (Chaiken et al., 1996; Petty and Cacioppo, 1986). And recent research found a strong correlation between use of Web assurance seals and user intention to use an online payment system (Ozkan, Bindusara and Hackney, 2010).

Web site social presence is a subjective quality based on user perception. It is defined as the perception of an interpersonal interaction due to the impression of human contact and the information richness of the medium (Gefen and Straub, 1997). Social presence features may include photographs of smiling customer service representatives as well as online-chat. Although Wang and Emurian (2005) found "social cue design elements" (p. 49 ) to be less important in promoting trust than visual design and content design, Gefen and Straub (2004) found evidence that the perception of social presence increases trust in e-commerce.

\subsection{Social Influence}

Social influence, sometimes referred to as subjective norms, is frequently decomposed into relevant referent groups. For example, in research that examined the use of information technology (IT) in an organization setting, Taylor and Todd (1995) decomposed sources of social influence into three groups: peers, superiors, and subordinates. In the context of e-commerce, Limayem, Khalifa, and Frini (2000) decomposed sources of social influence into three groups (friends, family and media), finding the social influence of media and family to have an effect on online shopping. Hwang (2005) found all three dimensions of social influence (friends, family, media) to be significantly related to online trust, while Bhattacherjee (2000) found news reports, popular press and mass media to have a large effect on subjective norms leading to intention to accept e-commerce. 
The existing literature suggests that these three factors are influential components in the complex relationship that occurs between an individual and an unknown online vendor: trust in the Internet infrastructure, Web site features of institutional trust and social presence, and social influence. These factors form the framework for the research presented here.

\section{Research Objective and Hypotheses}

In view of the inherent insecurity of the Internet and user concerns for information privacy, a question that should interest organizations seeking to maximize investments in e-commerce is: What cues of institutional trust and social presence are effective in overcoming low trust in the Internet infrastructure and social/media influences to persuade first-time users to provide personal information so that online transactions are facilitated? Specifically, three research questions are addressed:

In the context of initial trust formation:

- Does trust in the Internet infrastructure affect user willingness to provide personal information online?

- Do Web site elements of institutional trust and social presence affect user willingness to provide personal information online?

- Does general social influence affect user willingness to provide personal information online?

The research model is presented in Figure 1.

Trust in the Internet infrastructure is defined as trust in the safety and integrity of the fundamental security measures used to protect personal information during online transactions (McKnight and Chervany, 2001). Influential Web site characteristics are defined as artifacts of institutional trust (e.g., links to privacy policies and symbols of trusted third parties), and elements of social presence (e.g., e-mail links, images of service representatives, and options to speak online with service representatives in real time). User susceptibility to social or interpersonal influence is defined as the tendency of persons to change their online information-giving behavior as a result of social pressure (McGuire, 1968). The dependent variable is willingness to provide personal information ranging from data perceived as low risk (i.e., name, email address) to data perceived as high risk (i.e., credit card number, social security number) (Miyazaki and Krishnamurthy, 2002).

\section{Research Methodology}

The research consisted of a $3 \times 3 \times 3$ between-subjects quasi-experiment designed to test the effects of (1) trust in the Internet infrastructure, (2) social influence, and (3) Web site features of institutional trust and social presence on user willingness to provide personal information. The context of the study was anticipated patronage of an unknown Web vendor that offered a desired product at an acceptable price. The subjects were undergraduate and graduate students, considered to be reasonable proxies for online shoppers based on age and education (Drennan, Mort and Previte, 2006; Mauldin and Arunachalam, 2002). A total of 628 survey responses were included in the final analysis.

Respondents were advised that the topic of the survey was "Using the Internet for Personal Business." Using an online instrument, subjects responded to questions that assessed trust in the Internet infrastructure and susceptibility to social influence before being assigned to a media treatment. Assignment to treatment groups was accomplished with alphabetic self-selection menus. That is, based on the first letter of the last name (using self-selection), subjects were assigned to one of three media conditions: positive, negative, or none. Then, based on the first letter of the first name (using self-selection), subjects were assigned to one of three Web site conditions: low-, moderate-, or high-level. According to Shadish, Cook and Campbell (2002), this procedure is quasi-experimental in that random assignment occurred by means of self-selection.

User trust in the Internet infrastructure was evaluated using measures adapted from previous research (Lee and Turban, 2001; McKnight, Choudhury and Kacmar, 2002; George, 2004). Following assignment to a media treatment, susceptibility to social influence was measured using scales developed and validated as part of this study. The media treatments were composites of positive or negative excerpts pertaining to the safety of the Internet (selected from national magazines or newspapers and government or non-profit online sources) presented as print media. To provide and control for source credibility, both messages were presented as an article in USA Today. Following assignment to a simulated Web site on which the type and number of elements that represent guarantees, institutional assurances of trustworthiness, and social presence were varied, the effect of these elements was evaluated using measures adapted from previous research (Miyazaki and Krishnamurthy, 2002). The simulated Web site created for this experiment was "product-neutral" in that it typified a "registration" page on which new users would provide personal information to learn more about a product or service. 


\section{Data Analyses}

Statistical analyses included descriptive statistics, univariate analyses of factors affecting trust in the Internet infrastructure and susceptibility to social influence, cross-tabulations and chi-square tests to evaluate differences across treatment groups, and correlational analyses among the predictor variables. Logistic regression models were constructed to examine main and interaction effects.

\subsection{Descriptive Statistics}

The respondents were fairly evenly split by gender (342 males, 286 females), mostly young (453 were 18 to 24 years of age, 175 were 25 and older), and racially diverse (371 White, 182 Black/African American, 75 other races).

The majority of respondents reported using the Internet extensively: 584 reported daily use; time spent on the Internet averaged 17 hours per week. The majority of respondents (449) reported having used the Internet for 7 years or more. Approximately one fourth of the respondents reported making an Internet purchase on a monthly basis, while slightly more than half reported making an Internet purchase a couple of times a year. Table 1 presents characteristics of the participants.

\subsection{Logistic Regression Analyses}

Based on results of chi-square tests and correlational analysis, potential predictors of willingness to provide information included demographic characteristics, trust in the Internet infrastructure, susceptibility to social influence, media treatment, and Web site treatment. This paper focuses on the results of the logistic regression analyses.

Logistic regression relates one or more continuous or categorical predictor variables to a dichotomous dependent variable by analyzing the logit or natural logarithm of the odds of the reference outcome, defined as $\mathrm{P}_{\mathrm{i}}$ (the probability of an event). If $\mathrm{P}_{\mathrm{i}}$ is the probability of a "Yes" response, then 1- $\mathrm{P}_{\mathrm{i}}$ is the probability of a "No" response.

The logit transformation occurs in two steps: First, the odds of the event are determined $\left(\mathrm{P}_{\mathrm{i}} / 1-\mathrm{P}_{\mathrm{i}}\right)$, then the natural logarithm (ln) of the odds is calculated (Pampel, 2000). It is the logit, or log(odds), that serves as the dependent variable in logistic regression.

$$
\log (\text { odds })=\operatorname{logit}\left(\mathrm{P}_{\mathrm{i}}\right)=\ln \left(\mathrm{P}_{\mathrm{i}} / 1-\mathrm{P}_{\mathrm{i}}\right)
$$

A simple logistic regression equation with independent variable $\mathrm{X}$ takes the form:

$$
\operatorname{logit}\left(\mathrm{P}_{\mathrm{i}}\right)=\mathrm{a}+\mathrm{b}_{1} \mathrm{X}
$$

For a continuous covariate, $b_{1}$ gives the change in the $\log \left(\right.$ odds) for an increase of one unit in X. For example, if $b_{1}$ $=.555$, this value is exponentiated to learn the $\log$ (odds) or odds ratio $=1.742$. (In SPSS output, this is seen as $\operatorname{Exp}(B)=1.742$.) The odds ratio minus one $(1.742-1)$ means a one-unit increase in $X$ results in a $74.2 \%$ increase in the outcome of the target dependent variable (i.e., a "Yes" response).

For a categorical covariate, $\mathrm{b}_{1}$ gives the extent to which the odds in favor of one outcome are raised when $\mathrm{X}$ is raised from the reference level to another level. For example, if $b_{1}=1.888$, this value is exponentiated to learn the odds ratio $=6.606$. (In SPSS output, this is seen as $\operatorname{Exp}(\mathrm{B})=6.606$.) This means the odds of saying "Yes" (the target outcome) for those who did not receive a media treatment (the reference group) are 6.6 times greater than for those who received the negative media treatment (the comparison group).

An odds ratio greater than 1.0 signifies a positive relationship between two variables, and an odds ratio of less than 1.0 signifies a negative or inverse relationship. An odds ratio of 1.0 means there is no relationship between a predictor and the outcome (Menard, 1995). When an odds ratio is a fraction (i.e., . 750$)$, the reciprocal $(1 / .750=$ 1.33 ) is interpreted such that the odds of saying "Yes" for those who received the negative media treatment (the comparison group) are 1.33 times greater than for those who did not receive a media treatment (the reference group) (Pedhazur, 1997).

The logistic regression models were constructed using a model-building strategy (Hosmer and Lemeshow, 1989) that calls for univariate analysis of each variable to select variables for multivariate analyses with subsequent analyses considering interactions among the variables. Because chi-square statistics revealed significant differences in outcome between gender, race, media, and Web site groups, those variables were included in the initial regression model as were the continuous variables of interest (trust in the Internet, social influence of friends, social influence of family, and social influence of media). The results provided a subset of five covariates with $p$ $<.10$ that were retained for further analysis: race, trust, media, site, and social influence of family. The results of the reduced model showed these five variables to be significant at the .05 level for at least one outcome variable 
(phone number, credit card number, social security number) or all six outcome variables. Table 2 contains the results of the reduced multivariate model.

Ten two-way interactions may be formed from the variables in the reduced multivariate model. Following the strategy suggested by Hosmer and Lemeshow (1989), further analyses examined each of these interactions with all variables retained from the reduced multivariate model. When all outcome variables are considered collectively, none of the interaction models provides a significant improvement over the main effects only model. Therefore, the main effects model was selected for further analysis using the subset of predictor variables identified as significant for willingness to provide credit card number. Those covariates are race, trust, media, site, and social influence of family. This model was selected because it shares the highest level of significance (.000) with the model identified for willingness to provide address, and it is the most inclusive model; that is, it includes all variables that are significant for the remaining outcome variables. Of the five parameters in the final model, four were statistically significant. The estimates of the main effects logistic regression model are presented in Table 3.

The most frequently used test of significance of an individual predictor is the Wald Chi-square statistic (Pampel, 2000). This value indicates the relative importance of the individual variable. The estimates shown in Table 3 indicate four covariates in the model are important factors for willingness to provide personal information on the Internet in the context of initial trust formation.

Continuous variables. The $\operatorname{Exp}(\mathrm{B})$ value or odds ratio value for trust (1.463) indicates a one-unit increase in trust results in a $46.3 \%$ increase in the odds of the subject providing a credit card number. (Trust in the Internet infrastructure ranges in value from -3 to +3 in increments of 0.25 .) Based on a negative coefficient and a fractional odds ratio, using the reciprocal, the .868 odds ratio for social_ 2 indicates a one-unit increase in social influence of family results in a $15.2 \%$ decrease in the odds that the subject will provide a credit card number. (Social influence ranges in value from -3 to +3 in increments of 1.0.)

Categorical variables. The reference group for race is White; and race(1) compares Asian subjects to the reference group; race(2) compares Native Hispanic subjects to the reference group; race(3) compares Black/African American subjects to the reference group. Based on a negative B-value and a fractional odds ratio, using the reciprocal, the 3.67 odds ratio for race(1) indicates an Asian subject is 2.72 times less likely to provide a credit card number compared to a White subject (the reference group).

The reference group for site is low-level; and site(1) compares the high-level treatment to the low-level treatment; site(2) compares the moderate-level treatment to the low-level treatment. The odds ratio for site(1) indicates a subject who receives a high-level site treatment is 1.6 times more likely to provide a credit card number than a subject who receives a low-level site treatment. The odds ratio for site(2) indicates a subject who receives a moderate-level site treatment is 1.7 times more likely to provide a credit card number than a subject who receives a low-level site treatment.

\subsection{The Prediction Model}

A logistic regression classification table shows the overall success rate in predicting the outcome (yes or no). The overall accuracy of the reduced multivariate model to predict willingness to provide credit card number is $69.4 \%$. The positive predictive value $=55 / 88=62.5 \%$; the negative predictive value $=370 / 525=70.6 \%$ (Pedhazur, 1997) . The classification table for the logistic regression equation for estimating willingness to provide credit card number is shown in Table 4.

The equation for calculating the probability that a subject will provide a credit card number is given by the equation:

$$
\text { Probability }(\text { Yes })=1 /\left(1+\mathrm{e}^{-\mathrm{z}}\right)
$$

where $\mathrm{z}=$ the logistic regression equation derived from Table 2. For this model

$\mathrm{Z}=-1.111-1.003 * \operatorname{race}(1)+.380 *$ trust $-.142 * \operatorname{social} \_2+.470 * \operatorname{site}(1)+.544 * \operatorname{site}(2)$

(Note: $\operatorname{Race}(1)=$ Asian; site $(1)=$ high-level; site $(2)=$ moderate-level.)

This prediction model can be used to calculate the probability of willingness to provide credit card number based on the subject's race, scores on trust in the Internet infrastructure and susceptibility to social influence of family, and the level of the Web site treatment (low, moderate, high) (Chan, 2004). Several examples of the utility of the prediction model are provided below. 


\subsubsection{Example 1}

Two subjects are presented with a moderate level Web site. Each subject scores 1.5 on trust in the Internet infrastructure and 1.0 on susceptibility to social influence of family. These scores indicate the subjects are moderately trusting of the Internet and consider the opinions of family members when making decisions about providing information online or making purchases on the Internet. The first subject is non-Asian; the second subject is Asian. For the first subject, indicator (dummy) coding for race $(1)=\operatorname{site}(1)=0$, coding for $\operatorname{site}(2)=1$, and the logistic regression equation is:

$\mathrm{z}=-1.111+.380 * 1.5-.142 * 1+.544 * 1=0.139$

$\mathrm{e}^{-\mathrm{z}}=1.149$

Probability $($ Yes $)=1 /(1+1.149)=.465$

indicating the non-Asian subject is somewhat unlikely to provide a credit number at the moderate-level Web site in the context of initial trust formation.

For the second subject, indicator coding for $\operatorname{site}(1)=0$, coding for race $(1)=\operatorname{site}(2)=1$; the logistic regression equation is:

$\mathrm{z}=-1.111-1.003 * 1+.380 * 1.5-.142 * 1+.544 * 1=-1.142$

$\mathrm{e}^{-\mathrm{z}}=3.133$

Probability $($ Yes $)=1 /(1+3.133)=.242$

indicating the Asian subject is unlikely to provide a credit card number at the moderate-level Web site in the context of initial trust formation. This comparison shows that holding constant all other factors of the model, an Asian subject is much less likely to provide a credit card number than a non-Asian subject.

\subsubsection{Example 2}

Three subjects are presented with a moderate-level Web site. Each subject scores 2.0 on trust in the Internet infrastructure. However, scores on susceptibility to social influence of family vary from -1 to +1 . The first subject's score on social influence of family is -1 , indicating a lack of consideration for the opinions of family members when making decisions about providing information online or making purchases on the Internet. For this subject, the logistic equation is:

$\mathrm{z}=-1.111+.380 * 2-.142 *-1+.544 * 1=-.335$

$\mathrm{e}^{-\mathrm{z}}=.715$

Probability $($ Yes $)=1 /(1+.715)=.583$

The second subject's score on social influence of family is zero, indicating a neutral stance on the consideration of the opinions of family members. The logistic equation for this subject is:

$\mathrm{z}=-1.111+.380 * 2-.142 * 0+.544 * 1=.193$

$\mathrm{e}^{-\mathrm{z}}=.825$

Probability $($ Yes $)=1 /(1+.825)=.548$

The third subject's score on social influence of family is +1 , indicating moderate consideration for the opinions of family members. The logistic equation for this subject is:

$\mathrm{z}=-1.111+.380 * 2-.142 * 1+.544 * 1=.051$

$\mathrm{e}^{-\mathrm{z}}=.950$

Probability $($ Yes $)=1 /(1+.715)=.513$

This comparison shows that holding constant race, trust in the Internet infrastructure, and site-level, increasing levels of susceptibility to social influence function to reduce the probability that subjects will provide a credit card number in the context of initial trust formation.

\subsubsection{Example 3}

Three subjects are compared who differ only on the basis of Web site viewed. These subjects are non-Asian, have moderately high scores on trust in the Internet infrastructure (score $=2.0)$ and low positive scores on susceptibility to social influence of family (score $=1.0$ ) indicating moderate consideration of the opinions of family members. The first subject viewed a low-level site, the second subject viewed a moderate-level site, and the third subject viewed a high-level site. 
For subject one (low-level site), the logistic regression equation is

$\mathrm{z}=-1.111+.380 * 2-.142 * 1=-.493$

$\mathrm{e}^{-\mathrm{z}}=1.637$

Probability $($ Yes $)=1 /(1+1.637)=.379$

For subject two (moderate-level site), the logistic regression equation is

$\mathrm{z}=-1.111+.380 * 2-.142 * 1+.544 * 1=.051$

$\mathrm{e}^{-\mathrm{z}}=.950$

Probability $($ Yes $)=1 /(1+.950)=.513$

For subject three (high-level site), the logistic regression equation is

$\mathrm{z}=-1.111+.380 * 2-.142 * 1+.470 * 1=-.023$

$\mathrm{e}^{-\mathrm{Z}}=1.023$

Probability $($ Yes $)=1 /(1+1.023)=.494$

This comparison shows that holding constant race, trust in the Internet infrastructure, and social influence of family, a moderate-level web site results in the highest probability that a subject is willing to provide a credit card number. A high-level site results in a slightly lower probability, and the low-level site produces the lowest probability that a subject is willing to provide a credit card number in the context of initial trust formation.

In summary, the main effects model predicts with $62.5 \%$ confidence that, in the context of initial trust formation, Asian subjects are less likely than non-Asian subjects to provide a credit card number; increasing levels of social influence of family result in reduced probabilities that subjects will provide a credit card number; and a moderate-level Web site treatment results in the highest probability that subjects will provide a credit card number.

\section{Discussion and Conclusion}

The results of this experiment indicate trust in the Internet infrastructure, the presence of Web site features of institutional trust, and susceptibility to the social influence of media are positively related to willingness to provide personal information online in the context of initial trust formation. Additionally, significant differences in online information-giving behavior were observed between ethnic groups.

Evidence of systematic cognitive processing (Chaiken and Eagly, 1983) was provided by results that found significant differences in willingness to provide information across media treatment groups such that subjects who received the positive media treatment were more willing to provide information than subjects who received the negative media treatment. Because the media treatment required reading an article and answering manipulation check questions, these results describe systematic or "deep" cognitive processing (Chaiken et al., 1996). Evidence of heuristic cognitive processing (Chaiken and Eagly, 1983) was provided by results that found the presence of influential Web site characteristics influenced willingness to provide personal information. In the context of initial trust formation, when the online firm is unknown to the user, symbols of trusted third parties may provide brand recognition (Palmer et al., 2000). Because Web site features of institutional trust and social presence are processed as cues, these results describe heuristic or "shallow" cognitive processing (Chaiken et al., 1996). The heuristic-systematic model suggests that cognitive processing modes may occur simultaneously when motivation or capacity or both are high, and both modes of processing may have an impact on judgment. The primary difference in these two paths to attitude change lies in the amount of analysis given to the issue under consideration.

The results of this study provide insight for organizations that seek to adopt a strategy to maximize trust for new online users at the same time that they minimize investment in e-commerce. The utility of the prediction model for identifying the relative importance of factors and predicting outcomes can guide investment on Web site features that are sufficient for the specific data collection needs of the organization.

Because the quasi-experiment simulated a potential information-giving situation for a product-neutral, unknown (un-branded) Web site, the results should be interpreted within that limiting context. Also, this quasi-experiment included only one operationalization each of the media treatment and the Web site. Because media treatments were presented as an article in USA Today and the Web site was a fictional corporation, threats to construct validity include mono-operation bias such that the constructs of media influence and Web site features of institutional trust and social presence may have been underrepresented. Additionally, a threat to construct validity results from using one method of measuring outcome variables (i.e., self-report). 
Although previous research found social presence to be effective in increasing trust in e-commerce (Gefen and Straub, 2004), this study found no evidence that Web site features of social presence increase user willingness to provide information. Given contradictory findings and anecdotal evidence that Web site social presence features continue to evolve with advances in multimedia technology, future research should explore the use of interactive social features such as live chat and other forms of online communication to learn how multimedia elements impact initial trust in e-commerce.

Regarding differences found in information-giving behavior between subjects who received the positive media treatment and those who received the negative media treatment and in consideration of marketing research that indicates two-sided advertising messages result in higher believability and greater purchase intentions (Golden and Alpert, 1987), future research on media influences could look at the effectiveness of Web site information features such as news links and/or blogs that present opposing media treatments to offset negative media influences.

\section{References}

Bhattacherjee, A. (2000). "Acceptance of E-Commerce Services: The Case of Electronic Brokerages." IEEE Transactions on Systems, Man, and Cybernetics - Part A: Systems and Humans. 30(4), 411-420.

BBB Accredited Business Seal. (2010). Retrieved February 25, 2010, from www.bbb.org.

Belanger, F., Hiller, J.S., and Smith, W.J. (2002). "Trustworthiness in electronic commerce: the role of privacy, security, and site attributes." Strategic Information Systems, 11, 245-270.

BizRate Store Ratings (2009). Retrieved February 25, 2010, from http:/www.bizrate.com/ratings_guide/guide/

Chaiken, S., and Eagly, A.H. (1983). "Communication Modality as a Determinant of Message Persuasiveness and Message Comprehensibility." Journal of Personality and Social Psychology, 45, 241-256.

Chaiken, S., Wood, W., and Eagly, A.H. (1996). Principles of Persuasion. In E.T. Higgins and A.W. Kruglanski (Eds.), Social Psychology. Handbook of Basic Principles (pp. 702-742). New York: The Guilford Press.

Chan, Y.H. (2004). “Biostatistics 202: Logistic Regression Analysis.” Singapore Medical Journal,45, 55-61.

Drennan, J., Mort, G.S., and Previte, J. (2006). "Privacy, Risk Perception, and Expert Online Behavior: An Exploratory Study of Household End Users." Journal of Organizational and End User Computing, 18(1), 1-22.

Fogg, B.J., Soohoo, C., and Danielson, D. (2002). “How Do People Evaluate a Web Site's Credibility?” Consumer

Reports WebWatch. Retrieved December 18, 2009, from http://www.consumerwebwatch.org/ dynamic/web-credibility-reports-evaluate-abstract.cfm.

Gefen, D., Karahana, E., and Straub, D.W. (2003). “Trust and TAM in Online Shopping.” MIS Quarterly, 27(1), 51-90.

Gefen, D., and Straub, D.W. (1997). "Gender differences in perception and adoption of E-mail: an extension to the technology acceptance model.” MIS Quarterly 21(4): 389-400.

Gefen, D., and Straub, D.W. (2004). "Consumer trust in B2C e-Commerce and the importance of social presence: experiments in e-Products and e-Services.” Omega: The International Journal of Management Science, 32(6), 407-424,.

George, J.F. (2002). "Influences on the intent to make Internet purchases. Internet Research,” 12(2), $165-180$.

George, J.F. (2004). “The Theory of Planned Behavior and Internet Purchasing." Internet Research, 14(3), 198-212.

GeoTrust, About Us. Retrieved February 26, 2010, from www.geotrust.com.

Golden, L., and Alpert, M. (1987). "Comparative Analysis of the Relative Effectiveness of One-and Two-Sided Communication for Contrasting Products." Journal of Advertising, 16, 18-25.

HackerSafe, About HackerSafe, 2010. Retrieved February 25, 2010, from www.hackersafe.com.

Hosmer, D.W., and Lemeshow, S. (1989). Applied Logistic Regression. New York: John Wiley and Sons.

Hwang, Y. (2005). “An Empirical Study of Online Trust and Consumer Behavior: Cultural Orientation, Social Norms, and Personal Innovativeness in Information Technology." Proceedings of the Twenty-sixth International Conference on Information Systems, June 25-28, Las Vegas, Nevada.

ITRC (Identity Theft Resource Center) (2010). “ITRC 2009 Breach List,” January 8, 2010. Retrieved January 19, 2010, http://www.idtheftcenter.org 
Jarvenpaa, S.L., Tractinsky, N., and Saarinen, L. (1999). "Consumer Trust in an Internet Store: A Cross-Cultural Validation." Journal of Computer Mediated Communication, 5(2).

Jarvenpaa, S.L., Tractinsky, N., and Vitale, M. (2000). "Consumer Trust in an Internet Store." Information Technology and Management, 1(1), 45-71.

Lee, M.K., and Turban, E. (2001). “A Trust Model for Consumer Internet Shopping.” International Journal of Electronic Commerce, 6(1), 75-91.

Limayem, M., Khalifa, M., and Frini, A. (2000).“What Makes Consumers Buy from Internet? A Longitudinal Study of Online Shopping." IEEE Transactions on Systems, Man, and Cybernetics - Part A: Systems and Humans, 30(4), 421-432.

Mauldin, E., and Arunachalam, V. (2002). "An Experimental Examination of Alternative Forms of Web Assurance for Business-to-Consumer e-Commerce." Journal of Information Systems, 16(1), 33-54.

McGuire, W.J. (1968). "Personality and Susceptibility to Social Influence.” In E.F. Borgatta and W.W. Lambert (Eds.), Handbook of Personality Theory and Research, 1130-1187. Chicago: Rand McNally and Company.

McKnight, D.H., and Chervany, N.L. (2001). "Conceptualizing Trust: A Typology and E-Commerce Customer Relationships Model.” Proceedings of the 34th Hawaii International Conference on System Sciences, January 3-6, Maui, Hawaii.

McKnight, D.H., Cummings, L.L., and Chervany, N.L. (1998). "Initial Trust Formation in New Organizational Relationships." Academy of Management Review. 23(3):472-490.

McKnight, D.H., Choudhury, C.J., and Kacmar, V. (2002). "Developing and Validating Trust Measures for e-Commerce: An Integrative Typology." Information Systems Research, 13(3), 334-359.

Menard, S. (1995). Applied Logistic Regression Analysis. Thousand Oaks: Sage Publications.

Miyazaki, A.D., and Krishnamurthy, S. (2002). "Internet Seals of Approval: Effects on Online Privacy Policies and Consumer Perceptions." Journal of Consumer Affairs, 36(1), 28-40.

Ozkan, S., Bindusara, G., and Hackney, R. (2010). "Facilitating the adoption of e-payment systems: theoretical constructs and empirical analysis." Journal of Enterprise Information Management, 23(3), 305-325.

Palmer, J.W., Bailey, J.P., Faraj, S., and Smith, R.H. (2000). "The Role of Intermediaries in the Development of Trust in the WWW: The Use and Prominence of Trusted Third Parties and Privacy Statements." Journal of Computer Mediated Communication, 5(3).

Pampel, F.C. (2000). Logistic Regression: A Primer. Thousand Oaks: Sage Publications.

Pedhazur, E.J. (1997). Multiple Regression in Behavioral Research. Explanation and Prediction. $3^{\text {rd }}$ Edition. Stamford: Thompson Learning.

Pennington, R., Wilcox, H.D., and Grover, V. (2003). "The Role of System Trust in Business-to-Consumer Transactions.” Journal of Management Information Systems, 20(3), 197-236.

Petty, R.E., and Cacioppo, J.T. (1986). Communication and Persuasion: Central and Peripheral Routes to Attitude Change. New York: Springer-Verlag.

Shadish, W.R., Cook, T.D., and Campbell, D.T. (2002). Experimental and Quasi-Experimental Designs for Generalized Causal Inference. Boston: Houghton Mifflin Company.

Smith, H.J., Milberg, S.J., and Burke, S.J. (1996). "Information Privacy: Measuring Individuals' Concerns about Organizational Practices.” MIS Quarterly, 20(2), 167-196.

SquareTrade, About Us, 2010. Retrieved February 26, 2010, from www.squaretrade.com.

Taylor, S., and Todd, P.A. (1995). "Understanding Information Technology Usage: A Test of Competing Models." Information Systems Research, 6(2), 144-176.

TrustE, About TrustE (n.d.). Retrieved February 25, 2010, from www.truste.com.

VeriSign, About VeriSign (2010). Retrieved February 25, 2010, from www.verisign.com.

Wang, Y.D., and Emurian, H.H. (2005). "Trust in E-Commerce: Consideration of Interface Design Factors." Journal of Electronic Commerce in Organizations, 3(4), 42-60.

WebAssured, About Us (n.d.). Retrieved February 26, 2010, from www.webassured.com. 
Table 1. Characteristics of survey respondents

\begin{tabular}{|lr|lr|}
\hline Age & & Years Online \\
\hline $18-24$ & $72 \%$ & 7 years or more & $72 \%$ \\
$25-35$ & $21 \%$ & 4 to 6 years & $26 \%$ \\
$35-44$ & $5 \%$ & 3 years or less & $2 \%$ \\
45 and over & $2 \%$ & & \\
\cline { 3 - 4 } & & Frequency of Internet Use \\
\hline Gender & & Daily & $93 \%$ \\
Male & $54 \%$ & Weekly & $6 \%$ \\
Female & $46 \%$ & Monthly or less & $1 \%$ \\
& & \multicolumn{2}{|l}{} \\
\hline Ethnicity & & Frequency of Online Purchase \\
\hline White & $59 \%$ & Couple of times per year & $54 \%$ \\
Black/African American & $29 \%$ & Monthly & $26 \%$ \\
Asian & $8 \%$ & Weekly & $7 \%$ \\
Native Hispanic & $2 \%$ & Daily & $1 \%$ \\
Other & $2 \%$ & & \\
\hline
\end{tabular}

Note: The final dataset includes 628 cases. The respondents were mostly young, racially diverse and evenly split by gender. Respondents reported frequent usage of the Internet and occasional online purchase activity.

Table 2. Results of Reduced Multivariate Model

\begin{tabular}{|lccl|}
\hline & Model chi-square $G_{\mathrm{m}}$ & Sig. of $G_{\mathrm{m}}$ & $\begin{array}{l}\text { Significant Variables in the Equation } \\
\text { (Wald statistic } p<.05)\end{array}$ \\
\hline Name & 26.574 & .002 & race, trust \\
\hline Email & 25.718 & .002 & race, trust, media \\
\hline Address & 31.863 & .000 & race, trust \\
\hline Phone & 18.750 & .027 & social_2 (family) \\
\hline CCN & 54.056 & .000 & race, trust, site, social_2 (family) \\
\hline SSN & 16.952 & .049 & media \\
\hline
\end{tabular}

The results of the reduced multivariate model shows five variables to be significant at the .05 level for at least one outcome variable.

Table 3. Logistic Regression Model: Variables in the Equation

\begin{tabular}{|lrrrrrr|}
\hline & B & SE & Wald & Df & Sig. & Exp(B) \\
\hline Race & & & 8.854 & 3 & .031 & \\
\hline Race(1) & -1.003 & .397 & 6.398 & 1 & .011 & .367 \\
\hline Race(2) & -.595 & .739 & .648 & 1 & .421 & .551 \\
\hline Race(3) & -.367 & .203 & 3.259 & 1 & .071 & .693 \\
\hline Trust & .380 & .082 & 21.306 & 1 & .000 & 1.463 \\
\hline Media & & & 12.353 & 2 & .002 & \\
\hline Media(1) & .409 & .217 & 3.541 & 1 & .060 & 1.506 \\
\hline Media(2) & -.392 & .216 & 3.282 & 1 & .070 & .676 \\
\hline Social_2 & -.142 & .054 & 6.862 & 1 & .009 & .868 \\
\hline Site & & & 6.702 & 2 & .035 & \\
\hline Site(1) & .470 & .227 & 4.227 & 1 & .039 & 1.601 \\
\hline Site(2) & .544 & .223 & 5.927 & 1 & .015 & 1.723 \\
\hline Constant & -1.111 & .242 & 21.082 & 1 & .000 & .329 \\
\hline
\end{tabular}

The estimates shown in Table 3 indicate four covariates in the model are important factors for willingness to provide personal information on the Internet in the context of initial trust formation. 
Table 4. Prediction Model Classification Table

\begin{tabular}{|ll|c|c|c|}
\hline \multirow{2}{*}{} & \multicolumn{3}{|c|}{ Predicted } \\
\cline { 3 - 4 } Observed & \multicolumn{2}{|c|}{ Willing to Provide CCN } & \multirow{2}{*}{$\begin{array}{c}\text { Percentage } \\
\text { Correct }\end{array}$} \\
\hline CCN & No & Yes & $91.8 \%$ \\
\hline \multicolumn{2}{|c|}{ Yes } & 154 & 33 & $26.3 \%$ \\
\hline \multicolumn{2}{|c|}{ Overall Percentage } & \multicolumn{3}{c}{$69.4 \%$} \\
\hline
\end{tabular}

A logistic regression classification table shows the overall success rate in predicting the outcome (yes or no). The positive predictive value $=$ $55 / 88=62.5 \%$; the negative predictive value $=370 / 525=70.6 \%$.

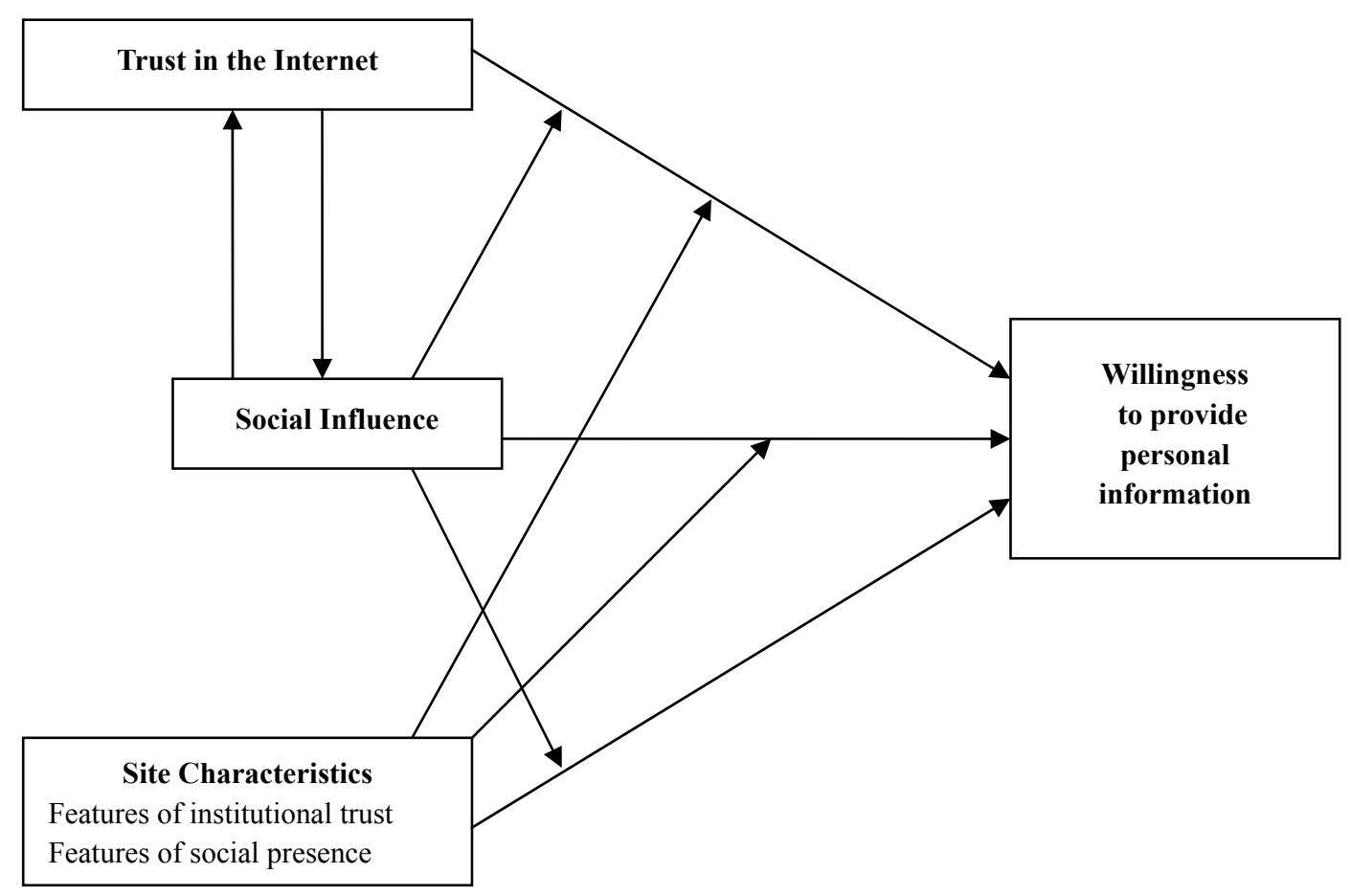

Figure 1. The Research Model 